\title{
Regulation of early signaling and gene expression in the $\alpha$-particle and bystander response of IMR-90 human fibroblasts
}

\author{
Shanaz A Ghandhi, Lihua Ming, Vladimir N Ivanov, Tom K Hei, Sally A Amundson*
}

\begin{abstract}
Background: The existence of a radiation bystander effect, in which non-irradiated cells respond to signals from irradiated cells, is well established. To understand early signaling and gene regulation in bystander cells, we used a bio-informatics approach, measuring global gene expression at 30 minutes and signaling pathways between 30 minutes and 4 hours after exposure to $\alpha$-particles in IMR-90 fibroblasts.

Methods: We used whole human genome microarrays and real time quantitative PCR to measure and validate gene expression. Microarray analysis was done using BRB-Array Tools; pathway and ontology analyses were done using Ingenuity Pathway Analysis and PANTHER, respectively. We studied signaling in irradiated and bystander cells using immunoblotting and semi-quantitative image analysis.

Results: Gene ontology suggested signal transduction and transcriptional regulation responding 30 minutes after treatment affected cell structure, motility and adhesion, and interleukin synthesis. We measured time-dependent expression of genes controlled by the NF- $\kappa$ B pathway; matrix metalloproteinases 1 and 3; chemokine ligands 2, 3 and 5 and interleukins $1 \beta, 6$ and 33. There was an increased response of this set of genes 30 minutes after treatment and another wave of induction at 4 hours. We investigated AKT-GSK3 $\beta$ signaling and found both AKT and GSK3 $\beta$ are hyper-phosphorylated 30 minutes after irradiation and this effect is maintained through 4 hours. In bystander cells, a similar response was seen with a delay of 30 minutes. We proposed a network model where the observed decrease in phosphorylation of $\beta$-catenin protein after GSK3 $\beta$ dependent inactivation can trigger target gene expression at later times after radiation exposure
\end{abstract}

Conclusions: These results are the first to show that the radiation induced bystander signal induces a widespread gene expression response at 30 minutes after treatment and these changes are accompanied by modification of signaling proteins in the PI3K-AKT-GSK3 $\beta$ pathway.

\section{Background}

Non-targeted effects could significantly enhance risks associated with exposure to low doses of ionizing radiation, which occurs in clinical and environmental contexts. It has been established that signals from irradiated cells travel through medium and cellular junctions to produce changes in gene expression [1,2], ROS production [3] and moderate damage to DNA in bystander cells as measured by micronucleus formation [4]. Although there is no direct epidemiological evidence for

\footnotetext{
* Correspondence: saa2108@columbia.edu

Center for Radiological Research, Columbia University, VC11-215, 630 West 168th Street, New York, NY, 10032, USA
}

these risks in humans, the potential importance of bystander effects is highlighted by the recent demonstration of radiation bystander carcinogenesis in a mouse model [5]. In primary fibroblasts the major players transmitting and maintaining signals between cells after irradiation appear to be soluble growth factors, cytokines, reactive oxygen species and extracellular matrix proteins [6,7]. A wealth of information exists on cellular events that occur 4 hours and later, including studies on gene expression $[1,2,8-10]$ cytokine production $[11], \gamma-$ H2AX measurement of DNA damage [12] and chromosomal end-points $[13,14]$ in directly irradiated cells and bystander cells. However, the events that precede these and other well-studied bystander effects on 
chromosomes [15] and DNA damage [13] are yet to be elucidated. In studies using $\gamma-\mathrm{H} 2 \mathrm{AX}$ as a marker of radiation induced DNA double strand breaks, the response in bystander cells was observed within 20-30 minutes after treatment [16]. A recent study confirmed a burst in cellular ROS levels 30 minutes after irradiation, followed by a 1-2 hr window during which double strand break repair foci were induced in $\alpha$-particle irradiated and bystander cells [17]. Other studies on signaling in bystanders have proposed that an early increase in production of reactive radicals [3] and TNFa [1] after irradiation can induce a cytokine cascade, which is consistent with the large number of signaling and stress response genes induced in this study.

In the present study, we focused on early responses to understand primary events that are more proximal to the bystander signal. 30 minutes after exposure, both irradiated and bystander cells showed a burst of gene expression changes. Gene ontology and pathway analyses of differentially expressed genes at 30 minutes after treatment suggested responses that affect cell structure and motility, signal transduction, transcriptional regulation and cell-to-cell communication. We validated the microarray results by quantitative real-time PCR and found that there was good concordance between these two methods. We were also interested in time-dependent patterns of gene expression and focused our studies on genes that showed induction at $30 \mathrm{~min}$ utes in both irradiated and bystander populations. The selected genes encode proteins that are transcriptional targets of NF- $\kappa \mathrm{B}$, and time course analysis of mRNA levels further supported our previous suggestion [2,3] that this signaling pathway is activated in bystanders in a synchronized manner from 30 minutes onward. From our earlier study that focused on the 4-hour transcriptional response [2], we predicted the involvement of $\beta$ catenin activation in gene expression in irradiated cells. We have now investigated protein modifications in the AKT-GSK3 $\beta$ signaling pathway upstream of $\beta$-catenin transcriptional activation. Our results show that the radiation signal can trigger a cascade of changes in AKT-GSK3 $\beta$ - $\beta$ catenin pathway almost concomitantly with a widespread gene expression response as early as 30 minutes after exposure.

\section{Methods}

\section{Cell culture, irradiation and RNA isolation}

Early passage (population doubling <35) IMR-90 human lung fibroblasts (Coriell repository, NJ) were sub-cultured in Dulbecco's modified Eagle's medium (Gibco) and Ham's F10 medium in a 1:1 mixture plus $15 \%$ fetal bovine serum. Mylar-bottomed culture dishes were prepared as described previously [1]. An inner dish with a base of 38-micron-thick Mylar strips was inserted into a larger dish with a 6-micron Mylar base. The 38-micron Mylar completely shields the alpha particles so that only cells on the thinner Mylar areas of the dish were directly irradiated. Cells seeded in these dishes formed a contiguous layer. Cells were exposed to 0 (sham irradiated) or $50 \mathrm{cGy}{ }^{4} \mathrm{He}$ ions (125 keV per micron) as simulated alpha particles using the track segment mode of the 5.5MV Singletron accelerator at the Radiological Research Accelerator Facility of Columbia University. This dose corresponds to an average fluence of approximately 6-12 alpha particles per cell in the irradiated sections of the dish and the probability of a cell not receiving a particle is less than $0.25 \%$. Four independent experiments were conducted.

Directly irradiated (outer dish) and bystander (inner dish) cells were separated at specified times after irradiation and RNA was isolated using Ribopure (Ambion, Life Technologies). RNA concentrations were measured using a NanoDrop ND-1000 spectrophotometer (Thermo Scientific) and RNA quality was monitored with the Agilent 2100 Bioanalyzer (Agilent Technologies, Santa Clara, CA). All RNA samples had RNA integrity numbers $>9.0$ [18] and $260 \mathrm{~nm} / 280 \mathrm{~nm}$ absorbance ratios $>2$.

\section{Protein isolation and Western blot procedure}

Directly irradiated (outer dish) and bystander (inner dish) cells were separated at specified times (30 minutes, 1 hour and 4 hours) after irradiation and trypsinized. For whole cell lysates, cells were collected, washed and lysed in 25\% glycerol, $40 \mathrm{mM}$ HEPES at $\mathrm{pH} 7.5,1 \mathrm{mM}$ DTT, $0.35 \mathrm{M} \mathrm{NaCl}, 0.5 \% \mathrm{NP}-40$ and Protease inhibitor mixture (HALT, Thermo Scientific). Separation of nuclear and cytoplasmic fractions was performed as recommended in the manufacturer's protocol for NucBuster $^{\mathrm{T}}{ }^{\mathrm{f}}$ from EMD Biosciences (Darmstadt, Germany). Protein concentrations were determined using the bicinchoninic acid method (Pierce) and measured using the Nanodrop-1000 spectrophotometer (Thermo Scientific). 50 micrograms of protein was used for western analysis and separated on $10 \%$ polyacrylamide gels. Primary antibodies were from Cell Signaling Technology, Boston: anti-AKT (cat\# 9272), antiphospho-AKT (S473) (cat\# 9271), anti-GSK3 $\beta$ (cat\# 9315), anti-phospho-GSK3 $\beta$ (S9) (cat \#9336), anti- $\beta$ catenin (cat\# 9562) and anti-phospho- $\beta$-catenin (S33/ 37/T41) (cat\# 9561). Other antibodies were purchased from Millipore: anti-actin (cat\# MAB1501) and Sigma: anti-TBP (cat\# T1827). Secondary antibodies were conjugated to horseradish peroxidase and signals were detected using enhanced chemi-luminescence (Amersham, GE). Relevant bands were quantified by densitometry using Image J, background corrected and normalized to actin levels, then compared to time matched controls. 


\section{Microarray Hybridization and Analysis}

RNA isolations were performed in parallel across irradiated, bystander and sham-irradiated samples, so that all samples were collected from one sub-cultivated pool of IMR-90 cells that were seeded from a single cryo-vial. After treatment, cells were lysed in pools from Mylar dishes at 30-minute, 1-hour, 2-hour, 4-hour, 6-hour and 24-hour time points. We repeated the experiment four times to provide four biological replicates. We analyzed the 30-minute RNA pools by microarray hybridization. Cyanine-3 (Сy3) labeled cRNA was prepared from 0.3 $\mu \mathrm{g}$ RNA using the One-Color Low RNA Input Linear Amplification PLUS kit (Agilent). Dye incorporation and cRNA yield were monitored with the NanoDrop ND1000 Spectrophotometer (Thermo Scientific). $1.5 \mu \mathrm{g}$ of cRNA ( $>9$ pmol Cy3 per $\mu$ g cRNA) was fragmented, hybridized to Agilent Whole Human Genome Oligo Microarrays (G4112F) using the Gene Expression Hybridization Kit, and washed following Agilent's recommendations. Slides were scanned with the Agilent DNA Microarray Scanner (G2505B). Default parameters of Feature Extraction Software 9.1 (Agilent) and grid version 014850_D_F_20090416 were used for image analysis, data extraction, background correction, and flagging of non-uniform features.

Background corrected intensities were $\log _{2}$ transformed and median-normalized in BRB-Array Tools, Version 3.8.0 [19]. Non-uniform outliers or features not significantly above background intensity in $40 \%$ or more of the hybridizations were filtered out, leaving 27,576 features. A further filter requiring a minimum 1.5-fold change in at least $20 \%$ of the hybridizations was then applied yielding a final set of 6911 features that were used for subsequent analyses. The microarray data are available through the Gene Expression Omnibus database using accession number GSE18760.

BRB-Array Tools was used to identify genes that were differentially expressed between controls and directly or bystander irradiated cells using a random-variance paired t-test, an improvement over the standard t-test that permits sharing information among genes about within-class variation without assuming that all genes have the same variance [20]. The test compares the differences in mean log-intensities between classes relative to the expected variation in mean differences computed from the independent samples. Genes with p-values less than 0.005 were considered statistically significant. The false discovery rate (FDR) was also estimated for each gene using the method of Benjamini and Hochberg [21], to control for false positives.

\section{Quantitative Real-Time PCR (qRT-PCR)}

The High-Capacity cDNA Archive Kit (Life Technologies, Foster City, CA) was used to prepare cDNA from total RNA. A custom low-density TaqMan array (Life Technologies, Foster City, CA) was designed using validated assays (additional file 1). Genes for inclusion on the low-density array (LDA) were selected on the basis of differential expression and low FDR, and seven previously selected endogenous control genes [2] were also included. For gene validation studies, $100 \mathrm{ng}$ cDNA was used as input for LDAs. Quantitative real time PCR reactions were performed with the ABI 7900 Real Time PCR System using Universal PCR Master Mix (Life Technologies), with initial activation at $50^{\circ} \mathrm{C}$ for 120 seconds and $94.5^{\circ} \mathrm{C}$ for 10 minutes followed by 40 cycles of $97^{\circ} \mathrm{C}$ for 30 seconds and $59.7^{\circ} \mathrm{C}$ for 60 seconds.

Relative fold-inductions were calculated by the $\Delta \Delta \mathrm{C}_{\mathrm{T}}$ method as previously used [22] using SDS version 2.3 software (Life Technologies). We applied geNorm [23] to the seven endogenous control genes on the LDAs to determine the most appropriate genes for normalizing the results. The LDA data was normalized to the geometric mean of peptidylprolyl isomerase A (PPIA) and ubiquitin $\mathrm{C}(U B C)$ gene expression levels.

\section{Gene ontology and pathway analysis}

The genes responding significantly $(\mathrm{p}<0.005)$ to either direct alpha particle or bystander irradiation were imported into DAVID, the database for annotation, visualization and integrated discovery http://david.abcc. ncifcrf.gov/[24]. The genes/proteins in our list were mapped to DAVID identifiers, and then functionally annotated using the DAVID biological processes and molecular function categories. The number of genes in each functional classification category was compared against the number of genes from the NCBI human genome in that category. The one-tailed Fisher exact $\mathrm{t}$ test probability value was used to statistically determine over- or under- representation of classification categories, Bonferroni corrected $\mathrm{p}$-values less than 0.05 were considered significant.

The sets of genes significantly responding to direct or bystander irradiation $(\mathrm{p}<0.005)$ were also imported into Ingenuity Pathways Analysis (IPA) (Ingenuity ${ }^{\circ}$ Systems, http://www.ingenuity.com) to analyze network interactions between the genes. The imported genes were mapped onto a global molecular network developed from information contained in the Ingenuity Pathways Knowledge Base. Networks of these significantly responding genes were then algorithmically generated based on their connectivity. The biological functions that were most significant to these networks were determined, and Fischer's exact test was used to calculate pvalues determining the probability that each biological function assigned to a network was due to chance alone. We also identified the IPA canonical pathways that were 
most significant within the differentially expressed gene sets.

\section{Results}

Early gene expression in irradiated and bystander cells In four independent experiments, RNA was extracted from control, directly irradiated, and bystander IMR-90 fibroblasts 30 minutes after exposure and was hybridized to Agilent Human whole genome arrays. Using the class comparison tool of BRB-Array Tools [19], we identified genes with significantly different expression compared with controls. In directly irradiated cells, 709 genes were differentially expressed ( $\mathrm{p}<0.005$ and false discovery rate $(\mathrm{FDR})<5 \%$; additional file 2 ). In bystander cells 407 genes were differentially expressed $(\mathrm{p}<0.005$ and FDR $<8.5 \%$; additional file 3). 293 genes responded significantly to both direct and bystander irradiation.

\section{Gene ontology analysis}

We then analyzed the differentially expressed gene lists from the microarray studies for enrichment of gene groups using the DAVID database [24]. The first step in this analysis was to map gene symbols to DAVID identifiers. In bystander cells, 292 DAVID identifiers were mapped from the list of 407 differentially expressed genes, and in the directly irradiated cells, 516 out of 709 differentially expressed genes mapped to DAVID identifiers. The most significant biological processes indicated in bystander cells were in categories related to protein modification ( $\mathrm{p}$-value $\left.7.6 \times 10^{-4}\right)$ and cell surface receptor mediated signal transduction (p-value $9.2 \times 10^{-3}$ ) (Table 1). Biological processes that were significant in the bystander response: protein modification, cell surface receptor mediated signal transduction, cell structure and motility, cation transport and ion transport; were also significant in $\alpha$-particle irradiated samples. The most significant category of differentially expressed genes in the irradiated condition was cation transport (p-value $6.2 \times 10^{-8}$ ), which was also significant in the bystanders ( $\mathrm{p}$-value $2.0 \times 10^{-2}$ ). Other significant categories in the directly irradiated cells, such as G-protein mediated signaling $\left(\mathrm{p}\right.$-value $\left.8.5 \times 10^{-4}\right)$ and cell surface receptor mediated signal transduction (p-value $4.4 \times$ $10^{-3}$ ) suggest a considerable involvement of activity at the cell membrane and inter-cellular signaling in the early response of directly irradiated cells, while the early response in bystanders was dominated by effects on signal transduction and cellular structure.

Examination of the molecular functions of significantly changed genes (Table 2) indicates that most of the early changes in gene expression in irradiated cells are related to cytoskeletal functions involving actin binding cytoskeletal protein ( $\mathrm{p}$-value $\left.2.1 \times 10^{-4}\right)$, suggesting that irradiation results in a rapid rearrangement of cell structure components. In bystanders, the most significantly affected molecular functions were non-receptor serine/ threonine protein kinases enzymes ( $\mathrm{p}$-value $8.2 \times 10^{-3}$ ) and cytoskeletal proteins (p-value $4.6 \times 10^{-2}$ ). As with biological processes, no significantly responding molecular functions at 30 minutes were unique among the bystanders.

\section{Quantitative real time RT-PCR validation of gene expression and time course analysis}

We selected 34 genes that were differentially expressed in both direct and bystander samples for validation using Taqman real-time polymerase chain reaction (qRT-PCR). We also performed qRT-PCR on the 30-

Table 1 Gene ontology analysis on biological processes

\begin{tabular}{lcc}
\hline PANTHER Biological Processes & BYSTANDER & IRRADIATED \\
\hline \hline BP00063:Protein modification & $7.63 \times 10^{-4}$ & $2.24 \times 10^{-2}$ \\
BP00103:Cell surface receptor mediated signal transduction & $9.22 \times 10^{-3}$ & $4.38 \times 10^{-3}$ \\
BP00285:Cell structure and motility & $1.05 \times 10^{-2}$ & $2.98 \times 10^{-4}$ \\
BP00143:Cation transport & $2.00 \times 10^{-2}$ & $6.16 \times 10^{-8}$ \\
BP00142:Ion transport & $2.00 \times 10^{-2}$ & $1.51 \times 10^{-2}$ \\
BP00044:mRNA transcription regulation & $\mathrm{NS}$ & $5.66 \times 10^{-7}$ \\
BP00071:Proteolysis & $\mathrm{NS}$ & $6.18 \times 10^{-4}$ \\
BP00104:G-protein mediated signaling & $\mathrm{NS}$ & $8.51 \times 10^{-4}$ \\
BP00020:Fatty acid metabolism & $\mathrm{NS}$ & $2.32 \times 10^{-3}$ \\
BP00067:Protein glycosylation & $\mathrm{NS}$ & $1.92 \times 10^{-2}$ \\
BP00292:Other carbon metabolism & $\mathrm{NS}$ & $2.66 \times 10^{-2}$ \\
BP00102:Signal transduction & $\mathrm{NS}$ & $2.84 \times 10^{-2}$ \\
BP00040:mRNA transcription & NS & $2.88 \times 10^{-2}$ \\
BP00064:Protein phosphorylation & NS & $3.00 \times 10^{-2}$ \\
BP00124:Cell adhesion & NS & $4.60 \times 10^{-2}$ \\
\hline
\end{tabular}

To identify significant PANTHER biological processes categories, $p$-values are Bonferroni corrected. NS is not significant $(p>0.05)$. 
Table 2 Gene ontology analysis on molecular functions

\begin{tabular}{|c|c|c|}
\hline PANTHER Molecular Functions & BYSTANDER & IRRADIATED \\
\hline $\begin{array}{l}\text { MF00213:Non-receptor serine/threonine } \\
\text { protein kinase }\end{array}$ & $8.20 \times 10^{-3}$ & $2.39 \times 10^{-2}$ \\
\hline $\begin{array}{l}\text { MF00261:Actin binding cytoskeletal } \\
\text { protein }\end{array}$ & $4.62 \times 10^{-2}$ & $2.05 \times 10^{-4}$ \\
\hline $\begin{array}{l}\text { MF00231:Microtubule binding motor } \\
\text { protein }\end{array}$ & NS & $3.23 \times 10^{-3}$ \\
\hline MF00091:Cytoskeletal protein & NS & $3.62 \times 10^{-3}$ \\
\hline MF00099:Small GTPase & NS & $4.49 \times 10^{-3}$ \\
\hline $\begin{array}{l}\text { MF00264:Microtubule family cytoskeletal } \\
\text { protein }\end{array}$ & NS & $2.70 \times 10^{-2}$ \\
\hline MF00224:KRAB box transcription factor & NS & $4.08 \times 10^{-2}$ \\
\hline MF00262:Non-motor actin binding protein & NS & $4.10 \times 10^{-2}$ \\
\hline
\end{tabular}

To identify significant PANTHER molecular function categories, $p$-values are Bonferroni corrected. NS is not significant $(p>0.05)$.

minute samples using 37 genes previously shown to change significantly at 4 hours [2], many (but not all) of which also responded in the 30-minute microarray data. The agreement between the two experimental approaches is very close, with a few exceptions where fold changes measured by qRT-PCR are higher than in microarrays (Figure 1). This effect has been observed previously by ourselves and others, and has been attributed to differences in probe choice and sequence specificity $[25,26]$. Some of the p53-regulated genes from the 4-hour responding set, such as CDKN1A, GADD45A, $F D X R, D D B 2$ and $F A S$, were relatively unchanged by both measurement approaches at the early 30 -minute time point, suggesting a delay in activation of the p53 pathway compared the cytokine/signal transduction effect in these cells [27]. In general, qRT-PCR analysis confirmed the up-regulation and down-regulation of a large number of mRNA at 30 minutes after treatment (Figure 1, additional files 2 and 3 ).

We used qRT-PCR to monitor the time dependent response of a subset of the genes that were induced early in both irradiated and bystander cells. Responses between 30 minutes and 24 hours were measured for NF- $\kappa \mathrm{B}$ responsive genes, such as matrix metalloproteinases 1 and 3 (MMP1 and $M M P 3)$, chemokine ligands 2, 3 and 5 (CXCL2, CXCL3 and CXCL5) and interleukins $1 \beta, 6$ and 33 (IL1B, IL6 and IL33). The results are summarized as a heatmap (Figure 2a), which clearly shows the biphasic pattern of expression in both irradiated and bystander samples. We show transcriptional profiles of three individual genes to illustrate this pattern of expression and synchronization of the gene response. Expression of IL6, MMP1 and CXCL5 (Figures $2 \mathrm{~b}, \mathrm{c}$ and $2 \mathrm{~d}$ ) in bystanders was nearly identical to that in irradiated cells suggesting that these NF- $\kappa$ B responsive genes were subject to very rapid and synchronous activation.

\section{Pathway analysis}

We then imported the lists of 709 and 407 genes from irradiated and bystander samples, respectively, into Ingenuity Pathway Analysis (IPA) to visualize pathway interactions of the responding genes/proteins. Top interacting networks of radiation responsive genes were significantly enriched for molecular and cellular functions of cell growth and proliferation $\left(\mathrm{p}=10^{-12}\right.$ to $\left.10^{-4}\right)$, cell death $\left(\mathrm{p}=10^{-12}\right.$ to $\left.10^{-4}\right)$ and cellular movement $(\mathrm{p}$ $=10^{-10}$ to $\left.10^{-4}\right)$. The top bystander networks were significantly enriched for cell death $\left(\mathrm{p}=10^{-13}\right.$ to $\left.10^{-4}\right)$, cell movement ( $\mathrm{p}=10^{-11}$ to $\left.10^{-4}\right)$ and cell-to-cell signaling $\left(\mathrm{p}=10^{-9}\right.$ to $\left.10^{-4}\right)$, additional file 4 . Pathway analysis of significant networks indicated that the NF- $\kappa \mathrm{B}$ transcription factor has a prominent role early in the bystander response. We also used IPA to connect gene networks to predict new regulatory hubs. Comparison of the 30minute responding genes with the 4-hour responding genes published previously [2], predicted a possible role for $\beta$-catenin protein as a transcriptional activator of gene expression at 4 hours. This pathway is modeled in Figure 3 using IPA and described in detail in the discussion. We reasoned that investigation of protein modifications in the $\beta$-catenin signaling pathway at early time points after treatment could help us to understand the fine tuning of the early response to radiation.

\section{Activation of AKT-GSK3 $\beta$ - $\beta$-catenin in bystander cells}

We focused on the AKT-GSK3 $\beta-\beta$-catenin axis of signaling in normal fibroblasts because pathway analysis predicted involvement of $\beta$-catenin as a transcriptional activator in the unfolding radiation bystander response. The results of western blot quantification of phosphorylated and basal protein levels of AKT kinase and GSK3 $\beta$ kinase are shown in Figure 4a, from 30 minutes to 4 hours after exposure. We observed an increase in the relative amount of activated AKT-P (Ser 473) in irradiated samples at 30 minutes, with consistent phosphorylation/activation of AKT through 4 hours post irradiation and a concomitant increase in GSK3 $\beta$ (Ser9) phosphorylation and inactivation. Bystanders appeared to lag behind the irradiated samples by an additional 30 minutes, showing increased levels of phosphorylated AKT in the 1 to 4 hour interval (Figure $4 \mathrm{a}$ and $4 \mathrm{c}$ ). In parallel with this, GSK3 $\beta$ phosphorylation was increased at 1 hour in bystanders. Inactivated by phosphorylation, GSK3 $\beta$ can further alter the phosphorylation status of $\beta$-catenin and we measured cytoplasmic $\beta$-catenin and phosphorylated $\beta$-catenin levels (Figure $4 \mathrm{~b}$ ). Decreasing levels of phosphorylated cytoplasmic $\beta$-catenin could be the result of increased GSK3 $\beta$ phosphorylation in both irradiated and bystander cells. The observed decrease in $\beta$-catenin phosphorylation and the accumulation of 


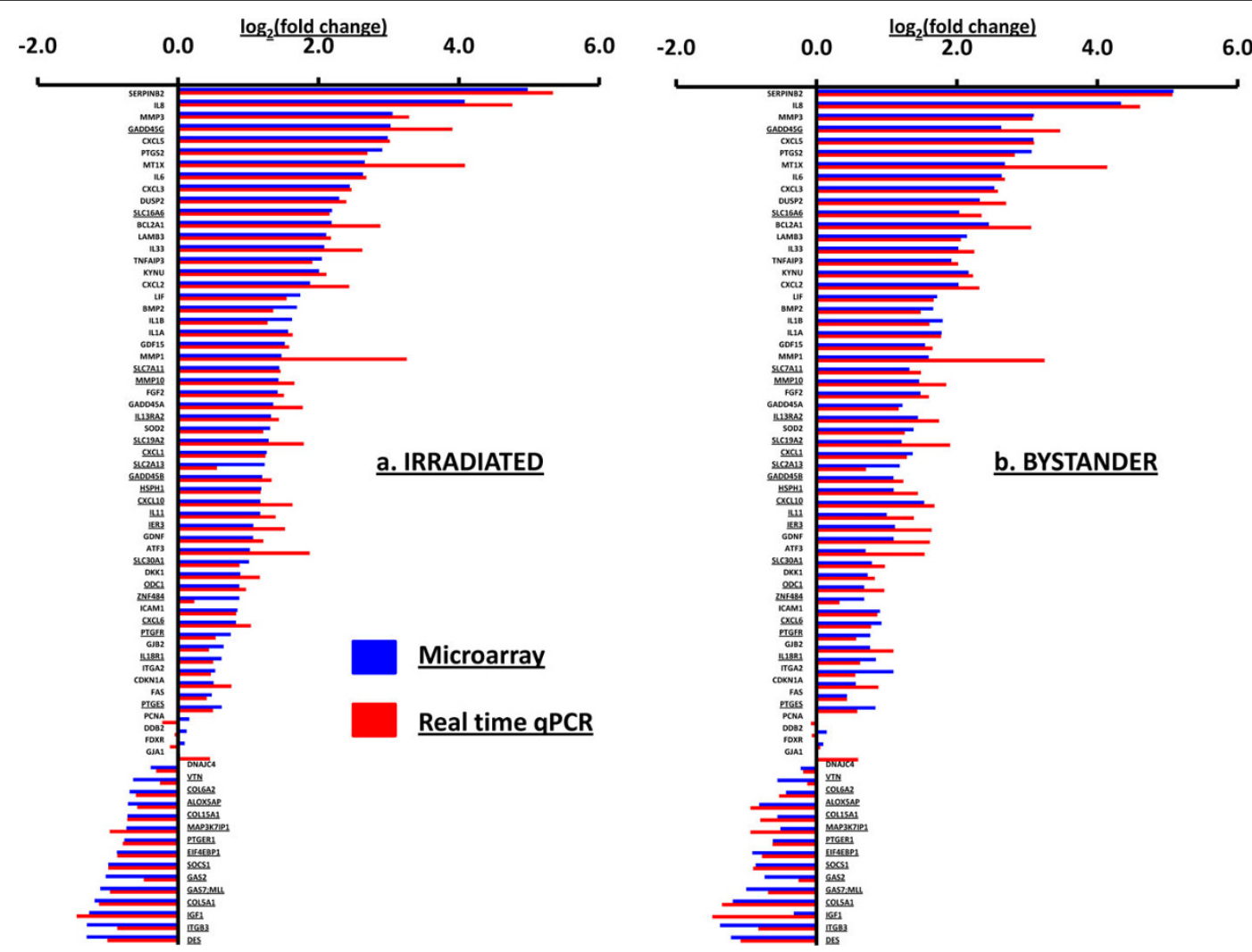

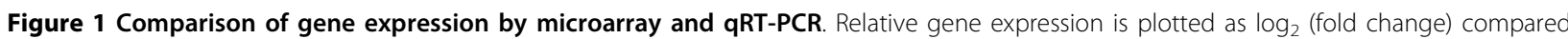
to matched sham-irradiated controls for irradiated (a) and bystander (b) samples at 0.5 hour after treatment. Each histogram is the mean of four biological replicates. Underlined genes are from class comparison results at 0.5 hour, the remaining genes were selected in our previous study [2] as responding at 4 hours. Microarray measurements (blue bars) and qRT-PCR measurements (red bars) are log 2 transformed ratios to controls; both up-regulated (right of the $y$-axis) and down-regulated genes (left of the $y$-axis) are shown.

$\beta$-catenin can lead to translocation to the nucleus where it may act as a transcriptional co-activator of gene expression of several genes such as PTGS2 and FAS.

\section{Discussion}

In this study we focused on early events to better understand the early signaling that shapes the radiation bystander effect. Time dependent analyses of specific genes such as PTGS2, IL8 and BCL2A1 had previously shown dramatic biphasic changes with a large response as early as 30 minutes after exposure [2]. This suggested that the re-programming of gene transcription is initiated prior to 4 hours after exposure. We also wanted to investigate signaling pathways in order to identify candidates for the upstream initiating signals that may lead to the gene expression changes observed at later time points. Therefore, we investigated the early burst of gene expression, looking for potential signaling nodes using a bioinformatics approach in conjunction with more conventional analyses of protein modifications of transduction pathways.
We measured global transcriptional changes at 30 minutes after treatment and found even more differentially expressed genes (709 in irradiated cells and 407 in bystanders) than we had previously reported at 4 hours, when 197 genes were changed significantly in irradiated cells and 137 genes were changed significantly in bystander cells [2]. The general patterns of gene expression were confirmed by qRT-PCR. Many genes that showed increases in expression after 4 hours were also induced at the 30-minute time in both irradiated cells (127 genes) and bystanders (120 genes). In contrast to the prominent role of p53 previously observed in the 4-hour response, however, there was no such pronounced p53 response at 30 minutes (Figure 1a) in the irradiated cells.

Of the 709 genes affected in directly irradiated cells, 416 genes were not significantly altered in bystanders. Gene ontology analysis using DAVID indicated that the top functional categories of the direct-exposure-only genes were in mRNA transcription regulation and activation of gene expression. These functions were not 


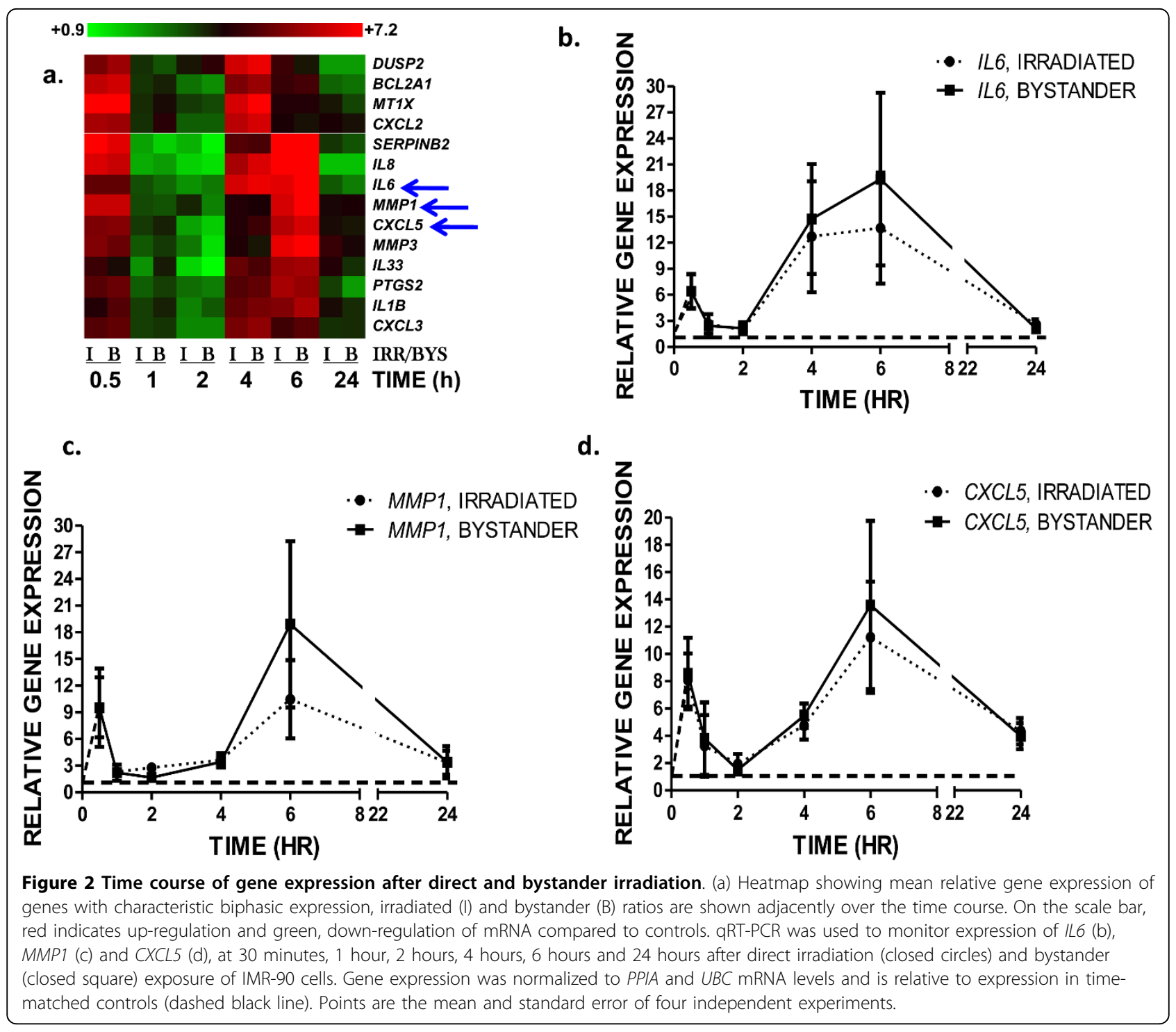

significant in bystanders at early times indicating that irradiation rapidly induced processes that altered the transcriptional landscape within hit cells, in addition to the overall stress response characterized by the release of cytokines and inflammatory regulators. The early bystander response was associated with cytokine signaling, cell structure changes and extra-cellular communication, suggesting that bystander fibroblasts were responding by altering their own cellular environment (Table 1). We had already reported that the gene expression responses of cyclooxygenase2 (PTGS2), interleukin-8 (IL8) and BCL2 related protein A1 (BCL2A1) were comparable at 30 minutes and 4 hours after exposure [2]. These genes are known targets for regulation by the NF- $\kappa \mathrm{B}$ transcription factor, and we predicted that the biphasic response in mRNA levels could be due to modulation by inhibitors of NF- $\kappa \mathrm{B}[28]$. In this study, we have verified that this was a frequent pattern of gene expression. Many genes that responded with an increase in mRNA levels at 4 hours showed a strong response at 30 minutes as well (Figures 1 and 2), suggesting coordinate regulation of genes with this biphasic pattern. These genes did not include canonical p53-response genes, which generally show peak induction at approximately 4 hours after irradiation, depending on the model system used. We further verified time dependent patterns of mRNA changes for 11 additional genes across the 24 hour time interval and found that they all showed peaks of induction after 30 minutes and 4 or 6 hours with a sharp decrease to control levels at the time between 1 and 2 hours, Figure 2a. This pattern of modular gene expression could be attributed to co- 


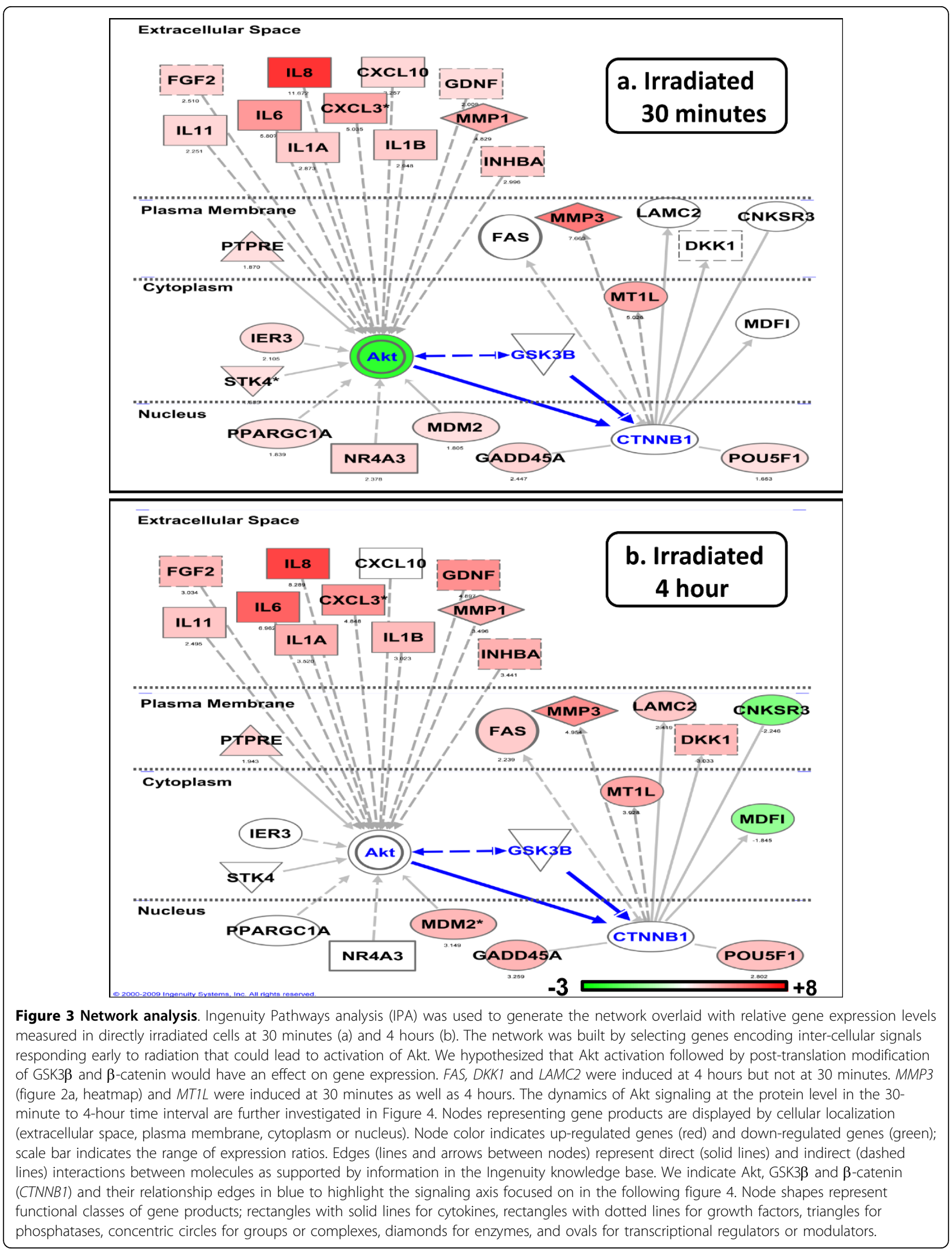




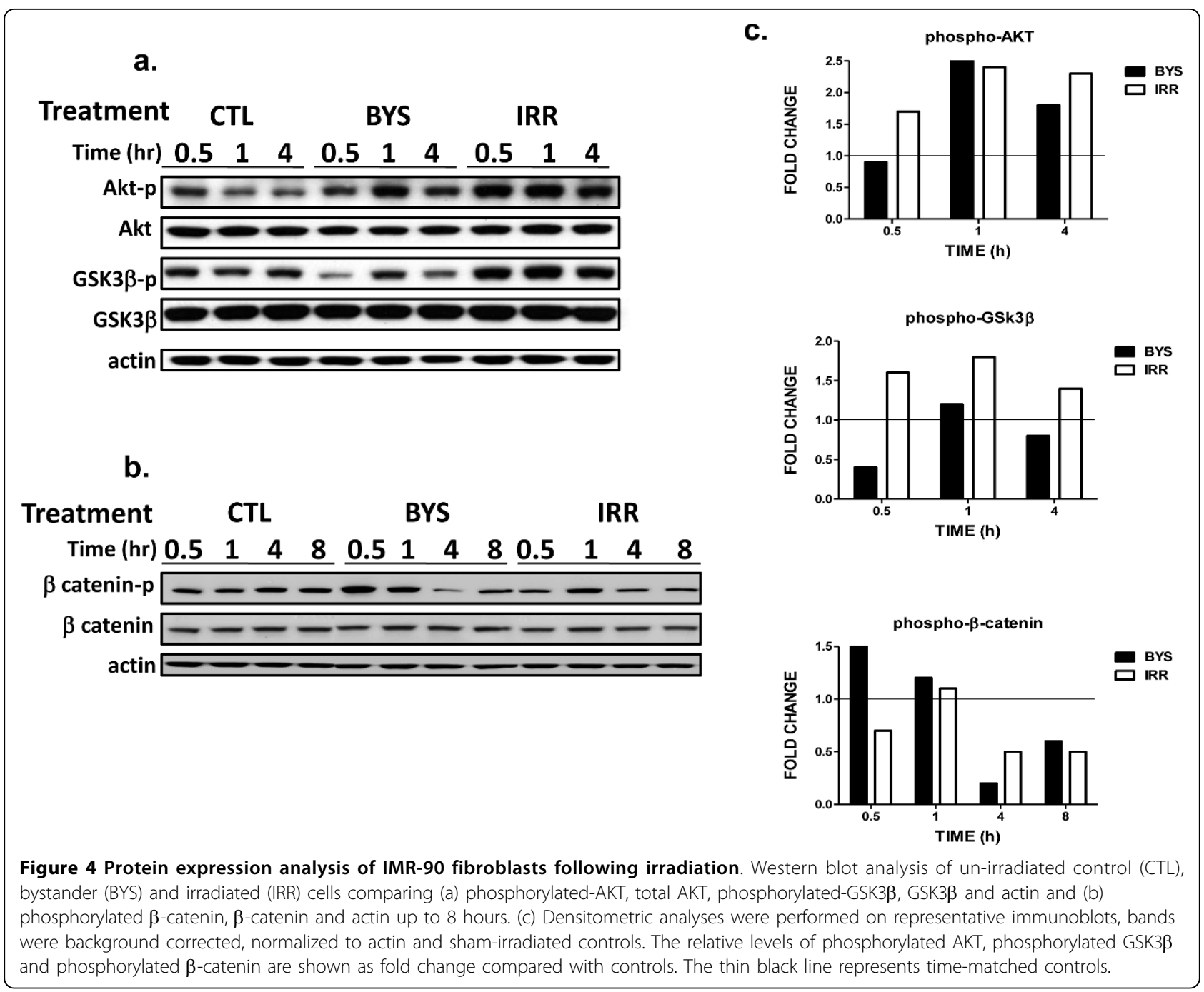

regulation of genes under the control of a transcription factor, such as NF- $\kappa \mathrm{B}$, acting as an early signal transducer $[3,29]$.

We compared the two gene sets, 709 genes in irradiated and 407 genes from bystander, for significant biological functions in IPA, which categorizes genes by mechanism- and disease-related associations. Gene expression changes in both irradiated and bystander cells appeared to be relatively similar in top-scoring biological functions categories such as cancer, cellular growth, proliferation, and cell death, which are very broad descriptions for functions associated with regulation of cell numbers and tissue homeostasis (additional file 4). 301 cancer-related genes in irradiated cells gave a p-value range from $1.2 \times 10^{-17}$ to $5.08 \times 10^{-4}$ for the cancer functional category. For 199 differentially expressed genes from bystanders; the $\mathrm{p}$-value range for the same category was $2.45 \times 10^{-16}$ to $2.36 \times 10^{-4}$. This indicated similar enrichment of this biological response in both gene sets. However, a closer look at the individual genes in the two conditions revealed differences, which were expected because direct irradiation would cause cells to have a more pronounced DNA damage and cell cycle response. IPA grouped 70 genes from 407 bystander responding genes under the functional category "cell-to-cell signaling" In IPA these genes were sub-grouped further by specific biological activities related to cell-to-cell signaling. We ranked these subcategories by decreasing order of number of genes affected and the highest ranked functions in bystander cells correlated with increases in activation, adhesion, communication, signaling, binding and stimulation of normal cells. From IPA and ontology analyses taken together, the mRNA changes suggest stimulation of cellular response in bystanders by signaling molecules as early as 30 minutes after treatment (additional file 4).

Pathway analysis of genes affected in irradiated cells after 4 hours suggested that in addition to p53 and NF- 
$\kappa \mathrm{B}$ transcription factors, $\beta$-catenin (most probably in concert with LEF1) could be an important transactivator of gene expression. We chose to focus on the AKT-GSK3 $\beta-\beta$-catenin axis of signaling in normal fibroblasts because of its central role in response to growth factors, changes in the medium, cytokine signaling, cell structure changes and stress due to production of reactive radicals, all of which are components of the bystander response [30]. Previous studies on bystander cells after carbon ion or alpha particle irradiation have also implicated activation of Akt signaling based on upregulation of target gene [9,31]. The activation of $\beta$ catenin as a nuclear activator of transcription is known to follow GSK3 $\beta$ phosphorylation by Wnt signaling or alternatively to be regulated through $\mathrm{PKB} / \mathrm{AKT}$ activation [30,32]. Cells responded to irradiation by rapid activation of $\mathrm{AKT}$ via phosphorylation and concomitant inactivation by phosphorylation of GSK3 $\beta$ at 1 to 4 hours. In bystanders, there was a similar pattern of GSK3 $\beta$ inactivation, but with a lag of 30 minutes or more. Cytoplasmic levels of phosphorylated $\beta$-catenin decreased gradually, which is considered a good indicator of trans-location to the nucleus [32] and activation of gene expression. This effect was also observed in bystanders, so $\beta$-catenin activity as a transcriptional coactivator of gene expression may occur in both populations. We have summarized these findings in Figure 3, which is a proposed pathway model of AKT-GSK3 $\beta-\beta$ catenin signaling pathway after irradiation. External signals such as growth factors, interleukins 6 and 8, which were induced early and secreted by irradiated cells could trigger activation of AKT in all cells [33] initiating the signaling cascade outlined in Figure 3, which results in gene expression activation of $\beta$-catenin target genes such as matrix metalloproteinase 3 (MMP3), Fas (FAS) and metallothionein 1L $(M T 1 L)$. In irradiated cells at 4 hours, this transcriptional regulator potentially regulated expression of $M T 1 L$ ( +3.9 fold change), DKK1 ( +3.0 fold change), MMP3 (+5.0 fold change), LAMC2 (+2.4 fold change) and $F A S(+2.3$ fold change) (Figure $3 \mathrm{~b})$. In bystander cells, only MMP3 ( +4.0 fold change) and $M T 1 L$ ( +3.8 fold change) were detected as differentially expressed at 4 hours. Of the genes predicted to be $\beta$ catenin targets, only $M M P 3$ showed the predicted NF$\kappa \mathrm{B}$ biphasic response that was similar in both irradiated and bystander cells. Although there is overlap and redundancy in the roles of both transcriptional regulators and target genes, our results suggest that this mechanism is active in both irradiated and bystander cells. The apparent lag between activation of this pathway in directly irradiated and bystander cells suggests that although it is clearly not the initiating signal, this pathway may play a role in the maintenance and later development of the bystander state. There is evidence for a lag in signaling between irradiated and bystander cells as observed by apoptosis induction in human fibroblasts [34], $\gamma \mathrm{H} 2 \mathrm{AX}$ foci induction after media transfer [35] and mutation induction in bystanders after treatment with conditioned media [36]. The mechanism driving the burst of cytokine and other gene expression seen in bystander cells at 30 minutes post-exposure has yet to be clearly elucidated.

We also observed some changes in AKT expression in our study. Although there was an overall increase in phosphorylation in both irradiated and bystander cells, total AKT protein levels were slightly decreased (Figure 4a). This could be a consequence of the significant down-regulation of $A K T 2$ mRNA observed at 30 minutes (0.4 \pm 0.1 fold change) (additional files 2 and 3 ) and 4 hours $(0.5 \pm 0.1$ fold change) in both irradiated cells and bystanders. The AKT antibody we used detected all three isoforms of AKT, and the phospho-Ser473-AKT antibody recognized phosphorylation of Ser473 on AKT1 as well as the corresponding residues in AKT2 and AKT3. However, GSK3 $\beta$ is known to be a substrate for all AKT isoforms [37], and in our system inactivation of GSK3 $\beta$ could result from activation of all AKT isoforms. Further studies will be required to distinguish the role of decreased $A K T 2$ mRNA specifically, and its contribution to phosphorylation and inactivation of GSK3 3 .

The importance of our study approach is that whole genome bio-informatics can help elucidate novel signaling networks that contribute to any phenotype. Exploring gene expression and signaling after irradiation led us to propose activation of an important signal transduction module, AKT signaling via $\beta$-catenin, as part of the cellular response in bystanders. Further verification of the role of $\beta$-catenin in bystanders will help us understand the complex nature of this response. In the field of radiation biology, there are few studies that use this approach, but a recent study on keratinocytes exposed to 1 cGy X-rays that identified the GATA3 transcriptional factor as a critical regulator of cellular radiation response at the genome level [38] is one example. Importantly, our study is the first to investigate the global transcriptional response within the first half hour after exposure with the goal of identifying signals more proximal to the generation of the bystander signal. This could have implications for the understanding of how cells respond in vivo and lead to understanding the rapid nature of the response.

\section{Conclusions}

A rapid and widespread transcriptional response occurs following irradiation in human fibroblast cells, and is communicated rapidly to bystanders. We found that cell structure and cell-cell communication processes are triggered very quickly in irradiated cells and these 
changes precede cell cycle gene responses that occur at a later time. Timing is important because release of free radicals and cytokines occurs quickly after irradiation and identifying early signaling events is critical to a full understanding of the range and extent of changes in bystander cells. The wave-like response of many genes following radiation suggests coordinate control of a large number of genes especially those that are controlled by NF $\kappa$ B. Activation of AKT-GSK3 $\beta-\beta$-catenin signaling early after irradiation and activation of target genes later in irradiated and bystander cells is a novel finding implicating a new pathway in bystander response. The connection between this signal transduction module, with the potential to converge extra-cellular communication with intra-cellular alterations of proteins and gene expression adds one more piece to the puzzle of stress response in a cell population where not all cells experience a direct ionization event.

\section{Additional material}

Additional file 1: PCR assay information.

Additional file 2: Class comparison of genes differentially expressed 30 minutes after direct irradiation.

Additional file 3: Class comparison of genes differentially expressed 30 minutes after bystander irradiation.

Additional file 4: Comparison of pathway analysis in irradiated and bystander cells at $\mathbf{3 0}$ minutes after irradiation.

\section{List of Abbreviations}

ROS: reactive oxygen species; TNF $\alpha$ : tumor necrosis factor alpha; NF- $\kappa$ B: nuclear factor kappa-light-chain-enhancer of activated B cells; AKT: v-akt murine thymoma viral oncogene homolog; GSK3 $\beta$ : glycogen synthase kinase 3 beta; BRB: Biometric research branch; FDR: false discovery rate; qRT-PCR: quantitative real time Reverse Transcription-Polymerase Chain Reaction; DAVID: The database for annotation, visualization and integrated discovery.

\section{Competing interests}

The authors declare that they have no competing interests.

\section{Authors' contributions}

SAG participated in the design of the study, carried out the microarray analyses and real-time PCR, and wrote the manuscript. LM carried out the protein analysis. $\mathrm{VI}$ contributed to the protein analysis and the writing of this manuscript. TKH contributed to the writing of this manuscript. SAA conceived of the study, participated in its design and data analysis and helped to draft the manuscript. All authors read and approved the final manuscript.

\section{Acknowledgements}

Analyses were performed using BRB-Array Tools developed by Dr. Richard Simon and Amy Peng Lam. We would like to thank Mr. Stephen Marino and the RARAF staff for assistance with irradiations. The Radiological Research Accelerator Facility (RARAF) is an NIH supported Resource Center through NIBIB grant EB-002033. This work was supported by NIH grant CA-49062.

Received: 22 March 2010 Accepted: 29 July 2010

Published: 29 July 2010
References

1. Zhou H, Ivanov VN, Gillespie J, Geard CR, Amundson SA, Brenner DJ, Yu Z, Lieberman HB, Hei TK: Mechanism of radiation-induced bystander effect: role of the cyclooxygenase-2 signaling pathway. Proc Natl Acad Sci USA 2005, 102(41):14641-14646.

2. Ghandhi SA, Yaghoubian B, Amundson SA: Global gene expression analyses of bystander and alpha particle irradiated normal human lung fibroblasts: synchronous and differential responses. BMC Med Genomics 2008, 1:63.

3. Zhou H, Ivanov VN, Lien YC, Davidson M, Hei TK: Mitochondrial function and nuclear factor-kappaB-mediated signaling in radiation-induced bystander effects. Cancer Res 2008, 68(7):2233-2240.

4. Prise KM, Belyakov OV, Folkard M, Michael BD: Studies of bystander effects in human fibroblasts using a charged particle microbeam. Int J Radiat Biol 1998, 74(6):793-798.

5. Mancuso M, Pasquali E, Leonardi S, Tanori M, Rebessi S, Di Majo V, Pazzaglia S, Toni MP, Pimpinella M, Covelli V, et al: Oncogenic bystander radiation effects in Patched heterozygous mouse cerebellum. Proc Natl Acad Sci USA 2008, 105(34):12445-12450.

6. Barcellos-Hoff MH, Park C, Wright EG: Radiation and the microenvironment - tumorigenesis and therapy. Nat Rev Cancer 2005, 5(11):867-875.

7. Barcellos-Hoff MH, Brooks AL: Extracellular signaling through the microenvironment: a hypothesis relating carcinogenesis, bystander effects, and genomic instability. Radiat Res 2001, 156(5 Pt 2):618-627.

8. Prise KM, O'Sullivan JM: Radiation-induced bystander signalling in cancer therapy. Nat Rev Cancer 2009, 9(5):351-360.

9. Iwakawa M, Hamada N, Imadome K, Funayama T, Sakashita T, Kobayashi Y, Imai T: Expression profiles are different in carbon ion-irradiated normal human fibroblasts and their bystander cells. Mutat Res 2008, 642(12):57-67.

10. Azzam El, de Toledo SM, Little JB: Expression of CONNEXIN43 is highly sensitive to ionizing radiation and other environmental stresses. Cancer Res 2003, 63(21):7128-7135.

11. Facoetti A, Mariotti L, Ballarini F, Bertolotti A, Nano R, Pasi F, Ranza E, Ottolenghi A: Experimental and theoretical analysis of cytokine release for the study of radiation-induced bystander effect. Int J Radiat Biol 2009, 85(8):690-699.

12. Dickey JS, Baird BJ, Redon CE, Sokolov MV, Sedelnikova OA, Bonner WM: Intercellular communication of cellular stress monitored by gammaH2AX induction. Carcinogenesis 2009, 30(10):1686-1695.

13. Hu B, Wu L, Han W, Zhang L, Chen S, Xu A, Hei TK, Yu Z: The time and spatial effects of bystander response in mammalian cells induced by low dose radiation. Carcinogenesis 2006, 27(2):245-251.

14. Ponnaiya B, Jenkins-Baker G, Bigelow A, Marino S, Geard CR: Detection of chromosomal instability in alpha-irradiated and bystander human fibroblasts. Mutat Res 2004, 568(1):41-48.

15. Hei TK, Zhou H, Ivanov VN, Hong M, Lieberman HB, Brenner DJ, Amundson SA, Geard CR: Mechanism of radiation-induced bystander effects: a unifying model. J Pharm Pharmacol 2008, 60(8):943-950.

16. Han W, Wu L, Hu B, Zhang L, Chen S, Bao L, Zhao Y, Xu A, Yu Z: The early and initiation processes of radiation-induced bystander effects involved in the induction of DNA double strand breaks in non-irradiated cultures. Br J Radiol 2007, 80(Spec No 1):S7-12.

17. Hanot M, Hoarau J, Carriere M, Angulo JF, Khodja H: Membranedependent bystander effect contributes to amplification of the response to alpha-particle irradiation in targeted and nontargeted cells. Int J Radiat Oncol Biol Phys 2009, 75(4):1247-1253.

18. Schroeder A, Mueller O, Stocker S, Salowsky R, Leiber M, Gassmann M, Lightfoot S, Menzel W, Granzow M, Ragg T: The RIN: an RNA integrity number for assigning integrity values to RNA measurements. BMC Mol Biol 2006, 7:3.

19. Simon R, Lam A, Li MC, Ngan M, Menenzes S, Zhao Y: Analysis of Gene Expression Data Using BRB-Array Tools. Cancer Inform 2007, 3:11-17.

20. Wright GW, Simon RM: A random variance model for detection of differential gene expression in small microarray experiments. Bioinformatics 2003, 19(18):2448-2455.

21. Benjamini $Y$, Hochberg Y: Controlling the False Discovery Rate - a Practical and Powerful Approach to Multiple Testing. Journal of the Royal Statistical Society Series B-Methodological 1995, 57(1):289-300. 
22. Amundson SA, Grace MB, McLeland CB, Epperly MW, Yeager A, Zhan Q, Greenberger JS, Fornace AJ Jr: Human in vivo radiation-induced biomarkers: gene expression changes in radiotherapy patients. Cancer Res 2004, 64(18):6368-6371.

23. Vandesompele J, De Preter K, Pattyn F, Poppe B, Van Roy N, De Paepe A, Speleman F: Accurate normalization of real-time quantitative RT-PCR data by geometric averaging of multiple internal control genes. Genome Biol 2002, 3(7):RESEARCH0034.

24. Huang DW, Sherman BT, Lempicki RA: Systematic and integrative analysis of large gene lists using DAVID bioinformatics resources. Nature Protocols 2009, 4(1):44-57.

25. Yuen T, Wurmbach E, Pfeffer RL, Ebersole BJ, Sealfon SC: Accuracy and calibration of commercial oligonucleotide and custom cDNA microarrays. Nucleic Acids Res 2002, 30(10):e48.

26. Draghici $S$, Khatri $P$, Eklund AC, Szallasi Z: Reliability and reproducibility issues in DNA microarray measurements. Trends Genet 2006, 22(2):101-109.

27. Amundson SA, Bittner M, Fornace AJ Jr: Functional genomics as a window on radiation stress signaling. Oncogene 2003, 22(37):5828-5833.

28. Hoffmann A, Levchenko A, Scott ML, Baltimore D: The IkappaB-NF-kappaB signaling module: temporal control and selective gene activation. Science 2002, 298(5596):1241-1245.

29. Eschrich S, Zhang H, Zhao H, Boulware D, Lee JH, Bloom G, Torres-Roca JF: Systems biology modeling of the radiation sensitivity network: a biomarker discovery platform. Int J Radiat Oncol Biol Phys 2009, 75(2):497-505

30. Franke TF: PI3K/Akt: getting it right matters. Oncogene 2008 27(50):6473-6488.

31. Ivanov VN, Zhou H, Ghandhi SA, Karasic TB, Yaghoubian B, Amundson SA, Hei TK: Radiation-induced bystander signaling pathways in human fibroblasts: a role for interleukin-33 in the signal transmission. Cell Signal 2010, 22(7):1076-1087

32. Gurung A, Uddin F, Hill RP, Ferguson PC, Alman BA: Beta-catenin is a mediator of the response of fibroblasts to irradiation. Am J Pathol 2009 174(1):248-255.

33. Datta SR, Brunet A, Greenberg ME: Cellular survival: a play in three Akts. Genes Dev 1999, 13(22):2905-2927.

34. Hamada N, Ni M, Funayama T, Sakashita T, Kobayashi Y: Temporally distinct response of irradiated normal human fibroblasts and their bystander cells to energetic heavy ions. Mutat Res 2008, 639(1-2):35-44.

35. Sokolov MV, Smilenov LB, Hall EJ, Panyutin IG, Bonner WM, Sedelnikova OA: lonizing radiation induces DNA double-strand breaks in bystander primary human fibroblasts. Oncogene 2005, 24(49):7257-7265.

36. Zhang Y, Zhou J, Baldwin J, Held KD, Prise KM, Redmond RW, Liber HL: lonizing radiation-induced bystander mutagenesis and adaptation: Quantitative and temporal aspects. Mutat Res 2009.

37. Stambolic V, Woodgett JR: Functional distinctions of protein kinase B/Akt isoforms defined by their influence on cell migration. Trends Cell Biol 2006, 16(9):461-466.

38. Bonin F, Molina M, Malet C, Ginestet C, Berthier-Vergnes O, Martin MT, Lamartine J: GATA3 is a master regulator of the transcriptional response to low-dose ionizing radiation in human keratinocytes. BMC Genomics 2009, 10:417.

\section{Pre-publication history}

The pre-publication history for this paper can be accessed here: http://www.biomedcentral.com/1755-8794/3/31/prepub

doi:10.1186/1755-8794-3-31

Cite this article as: Ghandhi et al:: Regulation of early signaling and gene expression in the $\alpha$-particle and bystander response of IMR-90 human fibroblasts. BMC Medical Genomics 2010 3:31.

\section{Submit your next manuscript to BioMed Central and take full advantage of:}

- Convenient online submission

- Thorough peer review

- No space constraints or color figure charges

- Immediate publication on acceptance

- Inclusion in PubMed, CAS, Scopus and Google Scholar

- Research which is freely available for redistribution

Submit your manuscript at www.biomedcentral.com/submit
C) Biomed Central 